\title{
Lifetime Analysis on Centrifugal ID Fan Foundation in Cement Plants
}

\author{
Hamid Eskandari-Naddaf ${ }^{1 *}$, Morteza Gharouni-Nik ${ }^{2}$, Amir Pakzad $^{1}$ \\ ${ }^{1}$ Department of Civil Engineering, Hakim Sabzevari University, Sabzevar, Iran \\ ${ }^{2}$ Department of Railway Infrastructure, School of Railway Engineering, Iran University of Science \& \\ Technology (IUST)
}

Received 10 October 2017; accepted 15 March 2018, available online 01 May 2018

\begin{abstract}
The dynamic behavior of centrifugal ID fan foundation of cement plants to perform the lifetime analysis was investigated in this study. To this end, a three-dimensional (3D) finite-element model (FEM) of this foundation was proposed and the analyses under static and dynamic loads for various modulus of elasticity of concrete $(E c=20,25,28$ and $30 \mathrm{GPa})$ and reinforcement $(E s=200,250,300 \mathrm{GPa})$ and rotor speeds of 400, 800, 1200 , and 1800 rounds per minute (rpm) were conducted. The results show that the stress and deflection for a certain ID fan is around 2.5 and 10 times more under dynamic loading compared to static loading. Therefore, the excessive vibrations induced by increased speed of ID fan can result in reduced lifetime and fatigue of the foundation. Comparison of FEM results with laboratory-based evidence verified the accuracy of simulations made by the FEM. The proposed model can thus be applied to determine the critical points in foundation structure in order to obtain optimum results of design parameters.
\end{abstract}

Keywords: Concrete Foundation, Compressive Strength, Loading, Modeling

\section{Introduction}

In the beginning of the twentieth century, the analysis of large concrete foundations was limited to static calculations based on vertical loads comprising the dead load plus 3-5 times of machine weight. However, it is now obvious that such designs with the first order natural frequency alone are not sufficient to characterize the dynamic behavior of large concrete foundations. In other words, a better understanding of the involved processes requires a dynamic analysis [1]. The serious challenges posed by increasing heights of towers and foundations along with concerns about design concepts, life cycle, environmental impacts, and dynamic load necessitate the revision of the existing production and assembling solutions [2].

The analytical methods like finite element analysis (FEA) are thus beneficial methods to include all parameters without the construction of full-scale structures [3, 4]. Research on FEM has shown that the first and second natural frequencies obtained from a stiffness matrix with coupled lateral behavior provided very good correspondence with the FEM of the foundation, especially when the effects of inertia on the foundation were negligible [5].

Operating deflection shape (ODS) models are also applied to identify the weaknesses of the foundation at a large ID fan. These models have indicated that adding mass and stiffness with more piles and concrete could be reliable except when resonance is involved. The reliability of such models can be obtained from Newton's second law of motion $(\mathrm{F}=\mathrm{m} \times \mathrm{a})$ which implies that the acceleration levels (a) would generally be reduced by increasing the mass $(\mathrm{m})$. Hooke's law $(\mathrm{F}=\mathrm{k} \times \mathrm{x})$, on the other hand, suggests that increased stiffness $(\mathrm{k})$ is generally associated with lower displacement levels (x) [6]. Therefore, isolation systems can be useful to reduce foundation vibration [7-9]. And the resonant frequencies of the rotor and support system may cause very high amplitude vibration [10]. Despite of whole efforts were done to reduce damages of these types of structures, their lifetime is less than that of expected. Due to this fact, some studies were done to investigate this important parameter, assessing the life cycle of photovoltaic and wind power technologies [11].

Another study was done by Seo et al. [12] in which redundant systems consisting of units that alternate between operating and standby states periodically were analyzed. Moreover, some other studies were carried out to evaluate lifetime of concrete structures such as dams [13] and bridges [14, 15]. Recently, Eskandari et al. performed a study to analyze the foundation of centrifugal ID fans in cement plants in which they investigated various parameters influenced in design situations [16].

While several studies have assessed some structures, which are subjected to static or dynamic load [17], the lifetime analysis of ID fan foundation and also the deformation and stress caused by fan vibration and mass have not been studied. These factors are in turn affected 
by the shape and size of the foundation and the strength of the applied materials $[18,19]$.

As the prediction of foundation behavior under various conditions can improve its structural performance, the present work aimed to use the FEM to accurately estimate the behavior of the concrete foundation under dynamic loads with different materials. To this end, the Abaqus Unified FEA which is a finite element-based software and a powerful tool to investigate the behavior of concrete structures [20] was applied in this study. It is noteworthy that due to the possible imbalance of the fan, the load applied to the foundation is generally dynamic unless a damper or isolator is used. Therefore, this study tried to evaluate the structural behavior of fan foundation under various types of load, e.g. static and dynamic with rotor speed of 400, 800, 1200 , and 1800 rounds per minute (rpm). It also used different material strengths, e.g. compressive strength of concrete and reinforcement to determine the critical points of the foundation structures in terms of displacements and stresses imposed to predict the actual behavior of the structure and the likelihood of further damage.

\section{Life design based on fatigue}

Foundation analysis requires the proper consideration of machine loads, categorized as static and dynamic loads and those exerted during operation, provided by the manufacturers. The main static load is generally caused by the dead load of the equipment. On the other hand, the magnitude of the moments produced by the driving mechanism, typically calculated as a vertical force couple, depends on both the rotational speed and power output.

Imbalance, created when the rotating part's center of mass does not match the center of rotation, is responsible for major dynamic loads during operation. Although these loads are commonly presented by the machine manufacturer, they can also be computed based on the balance quality grade of the rotor.

The resultant imbalanced load $\mathrm{F}(\mathrm{t})(\mathrm{N})$ is calculated with the rotating mass $\mathrm{m}(\mathrm{kg})$ as follows [13]:

$$
\mathrm{F}(\mathrm{t})=\mathrm{me} \omega^{2}
$$

Where:

e $(\mathrm{mm})=$ permissible eccentricity and $\omega(\mathrm{rad} / \mathrm{s})=$ velocity of the rotor.

Since such an imbalance increases over the course of operation, when fixing it is for the analysis, the $F(t)$ obtained from Eq. (1) has to be multiplied by a factor, which should typically, but not always, be greater than 2 [13].

Dynamic analysis is performed based on the vibration modes of similar structures and the vibrations measured at various fan speeds (e.g. 400, 800, 1200, 1500, and $1800 \mathrm{rpm}$ ). According to Eq. (1), the total F (t) at all bolts, caused by fan rotation, would be respectively $10,20,30,40$, and 50 tons for the above-mentioned rotational frequencies. Based on the direction of fan rotation, the concentrated force should be considered tensile at one side and compressive at the other. The static load exerted on the surface by the weight of the equipment was considered 60 tons. The FEM model of ID fan foundation has been depicted in Fig. 1.

A three-dimensional (3D) numerical model (length $(7 \mathrm{~m})$, width $(3 \mathrm{~m})$ and depth $(3 \mathrm{~m}))$ was developed to investigate the behavior of the concrete foundations of ID fans under combined loading conditions.

Finite element programming was performed with Abaqus Unified FEA 6.13. The concrete components and steel bars were first generated by the software (based on actual practical details) and then assembled at their appropriate locations.

Proper meshing of the obtained structural components is critical to the accuracy of the results. Therefore, a 10node quadratic tetrahedron (C3D10) mesh and a 3-node quadratic beam in space (B32) mesh were considered for the concrete structure and the steel bars, respectively.

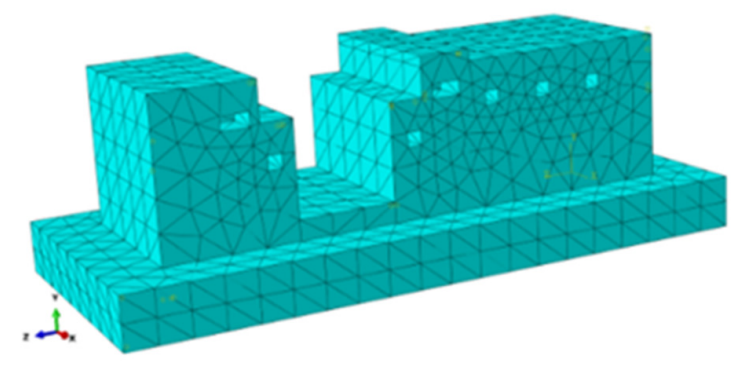

Fig. 1 ID Fan modeling in Abaqus software.

Dynamic stress is repetitive and can cause fatigue. Fatigue can be calculated by multiplying the dynamic stress by a fatigue factor. Since fatigue can damage the foundation of ID fans, the fatigue limit of the foundations needs to be accurately examined. The load combination associated with this condition is determined based on S-N curves describing the number of cycles $(\mathrm{N})$ for each amplitude reversal load (S). These curves are built on a yearly basis considering all possible load situations that may occur during operation and extrapolated for the lifetime of the foundation (usually 20 years). Fatigue life is defined as the number of cycles before failure. The fatigue life of concrete subjected to cyclic stresses may be calculated from [21]:

$$
\log _{10} N=C_{1} \frac{\left(1-\frac{\sigma_{\max }}{C_{5} \cdot f_{r d}}\right)}{\left(1-\frac{\sigma_{\min }}{C_{5} \cdot f_{r d}}\right)}
$$

Where:

$f_{r d}=$ the compression strength for the type of failure in question.

$\sigma_{\max }=$ the numerically largest compressive stress calculated as the average value within each stress-block. 
$\sigma_{\min }=$ the numerically least compressive stress calculated as the average value within each stress-block.

$c_{5}=$ fatigue strength parameter. For concrete $c_{5}$ shall be taken equal to 1 and $c_{1}=12$ for structures in air [21].

\section{Results and discussion}

Fatigue analysis can be helpful to understand intensification of micro cracks which are not reported till now. As mentioned earlier, ID fan foundations seem to be prone to fatigue damage and must thus be checked for fatigue limit. Dynamic stress is repetitive and can cause fatigue. Fatigue can be computed based on the static allowable load and by multiplying the dynamic stress by a fatigue factor. According to equation 1 and equation 2 for various speeds, the exerted point load will be compressive on one side of the pin and tensile on the other. The calculations were performed based on the number of loading and unloading cycles in one year. In order to determine the effects of material characteristics on foundation lifetime, 24 FEMs were analyzed. To simplify the analysis, the main points that would affect lifetime are plotted in Figs. 2 to 5 for $400 \mathrm{rpm}$ to 1800 $\mathrm{rpm}$. These frequencies are chosen based on their usage in ID Fans rotors [16]. It can be seen that material properties have direct effect on lifetime while number of rpms affects inversely. Moreover, considering various combinations including speed, Ec, and Es, the optimal material characteristics and speed to obtain greater performance, minimal construction costs, and maximum lifetime for foundations under dynamic load can be determined.

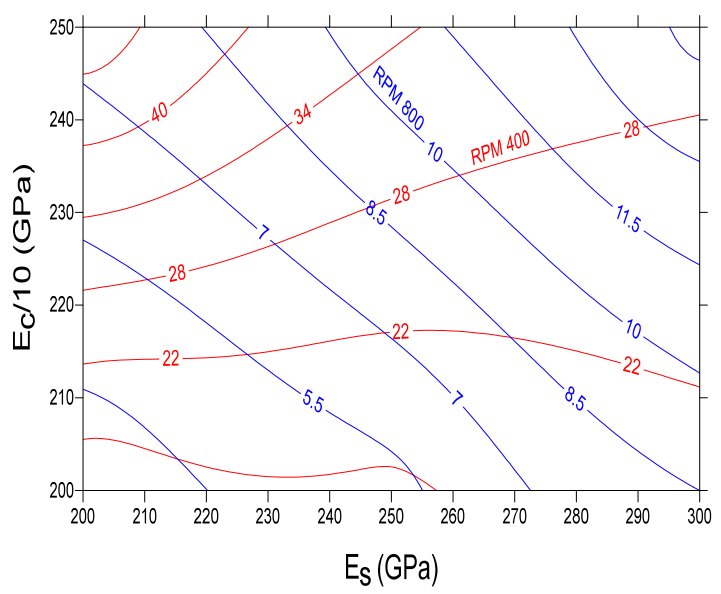

Fig. 2 Lifetime based on the (Ec), (Es), and rpm $=400$ and 800

As seen in Fig. 2, increased speed would be associated with decreased lifetime. It also indicates that by increasing in amount of Ec for $400 \mathrm{rpm}$, changes of Es are negligible which emphasizes the sensitivity of lifetime function on Ec value. This issue continues to 800 rpm but after that the trend is different so that the effects of both Ec and Es parameters on lifetime are obvious. Stress values in $400 \mathrm{rpm}$ is in linear phase but by enhancing the rotor speed these stresses increase also and lead to Fc value, thus the process arrived in nonlinear steps in stress-strain curve and then effect of steel mechanical properties like Es may be noticeable. By increasing the rotor speed to 1200 (Fig. 3), 1500 (Fig. 4) and finally 1800 (Fig. 5) the effect of Es gradually increases.

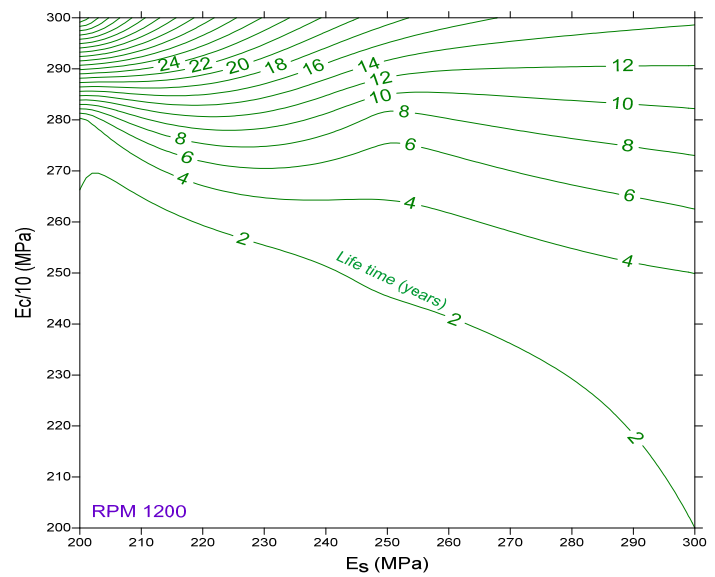

Fig. 3 Lifetime based on the (Ec), (Es), and $\mathrm{rpm}=1200$

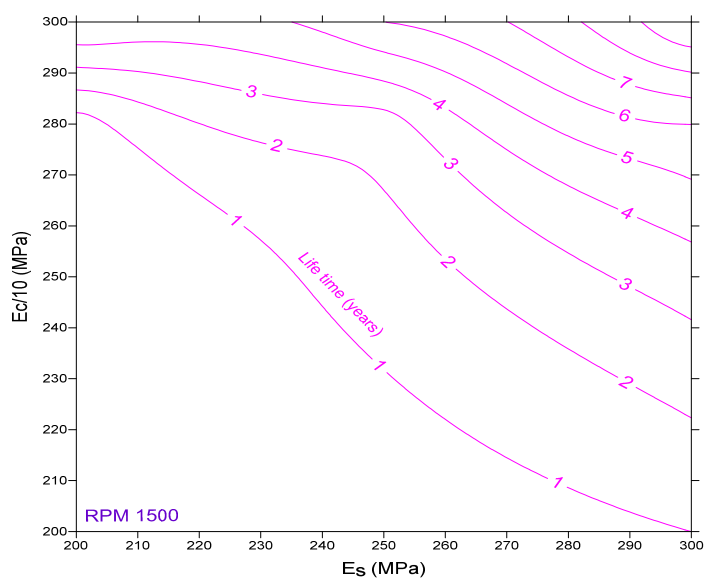

Fig. 4 Lifetime based on the (Ec), (Es), and rpm=1500

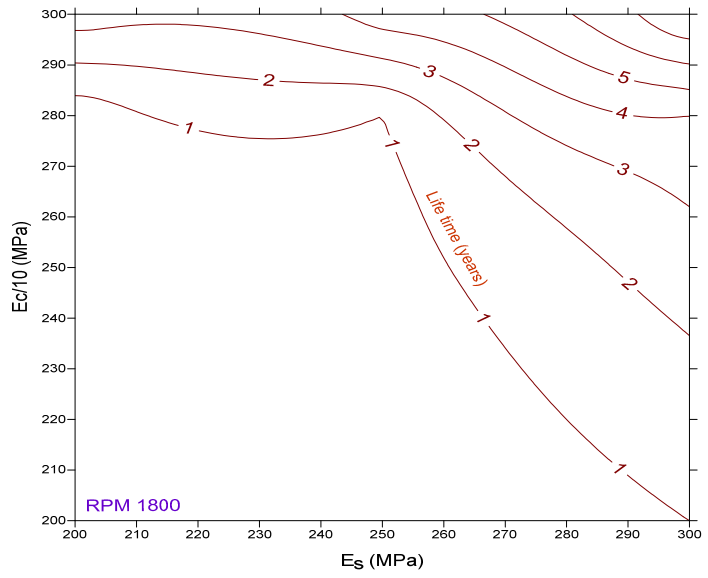

Fig. 5 Lifetime based on the (Ec), (Es), and rpm=1800 
The gradient of Es variations by enlarging the rotor speed is also increased, which infers the high sensitivity of lifetime function on Es value.

Considering various rotor speeds, it is so obvious that increasing of this parameter causes growth in stress values in foundation structure and then in moving the stress-strain curve from linear to nonlinear phase. Numerous load and unload cycles would induce fatigue damage increments which resulted in faster arrival into nonlinear phase. Therefore, compressive stress block is reduced faster which lead to growing the effect of steel mechanical properties such as Es on lifetime function of the foundation.

According to the above mentioned, it can be deducted that in lifetime function of the structure which is applied under dynamic loads, number of load-unload cycles, Es and Ec could have important influences. For cycles with low frequency the stresses are in elastic and elastic-plastic ranges where the lifetime function is more dependent on variations of Ec. Afterward, by enlarging the number of cycles which is followed by increased stresses, the sensitivity of lifetime function gradually moves to variations of Es.

Results of the analysis for Es $=200 \mathrm{GPa}$ and various amounts of Ec have been depicted in Figs. 6 to 9 for $\mathrm{Ec}=20,25,28$, and $30 \mathrm{GPa}$, respectively.

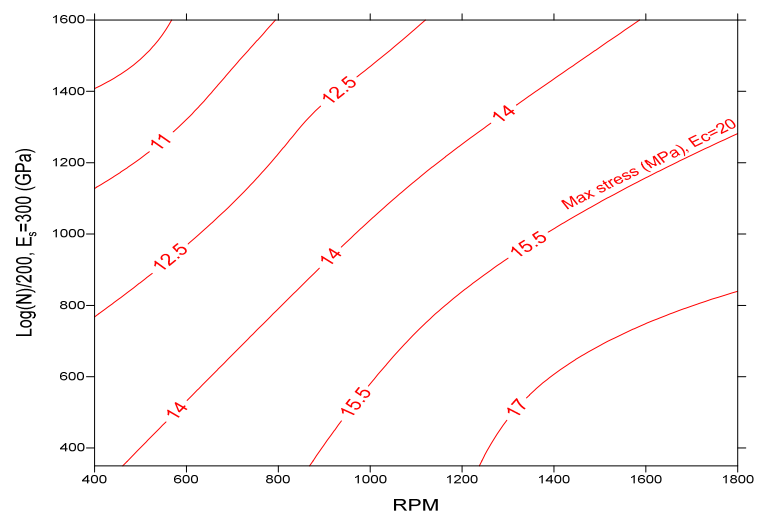

Fig. 6 Lifetime based on the speed and $\log (N)$ for $(E s)$ $=300$ and $\left(\mathrm{E}_{\mathrm{c}}\right)=20 \mathrm{GPa}$

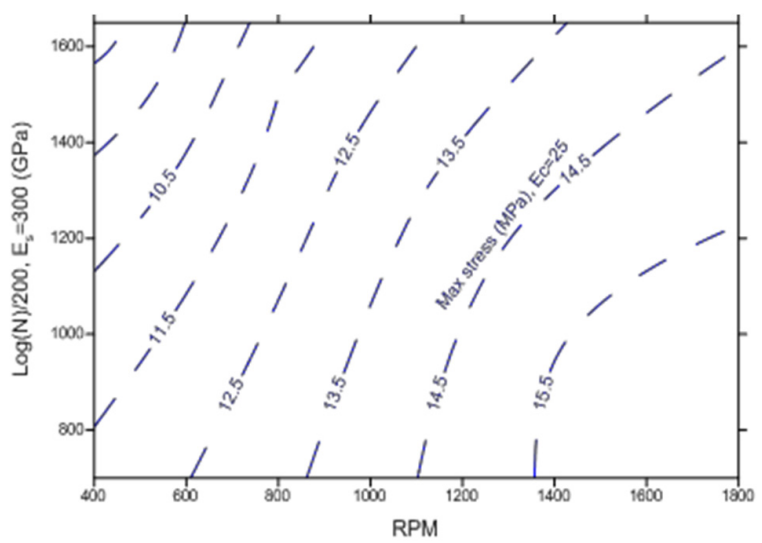

Fig. 7 Lifetime based on the speed and $\log (N)$ for $(E s)$ $=300$ and $(\mathrm{Ec})=25 \mathrm{GPa}$
Imposed stresses of the foundation structure could be increased by developing the rotor speed or load frequency as well as number of load-unload cycles so that the variation of rotor speed is more effective than that two others. As seen, utilizing higher values of Ec can cause more number of applied load - unload cycles to the structure with remaining in the linear phase.

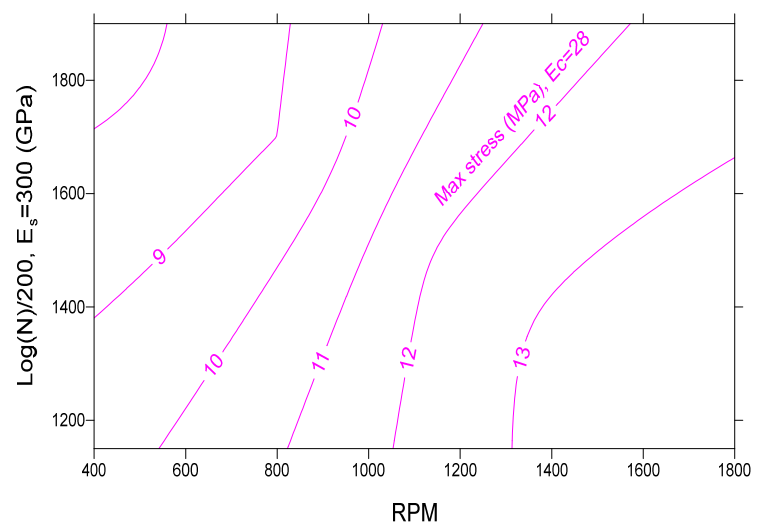

Fig. 8 Lifetime based on the speed and $\log (\mathrm{N})$ for (Es) $=300$ and $(\mathrm{Ec})=28 \mathrm{GPa}$

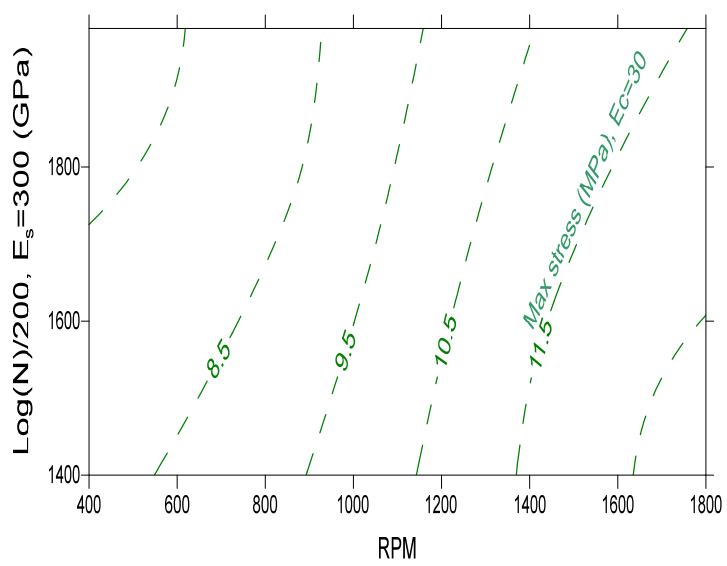

Fig. 9 Lifetime based on the speed and $\log (N)$ for (Es)

$$
=300 \text { and }(\mathrm{Ec})=30 \mathrm{GPa}
$$

For example, for $1400 \mathrm{rpm}$ and $\log (\mathrm{N}) / 200$ equal to 600 , the maximum amounts of stress for Ec of 20, 25, 28 and $30 \mathrm{GPa}$ are about 17, 16, 13 and $11.5 \mathrm{MPa}$ respectively. Similarly this kind of illustrations can be developed for other values of involved parameters to obtain optimum results of each situation.

\section{Conclusion}

Lifetime of ID fan foundations in cement plants is of utmost economic importance. The analysis, design, and implementation of this type of foundations should thus provide maximum confidence and operational safety. Developing FEMs for all parts, especially the foundation, of an ID fan can lead to better performance under static and dynamic loading. Such models can also determine the 
effects of various material characteristics on the stresses and deflections in all directions.

From the FEM, the stress and deflection for a certain ID fan is around 2.5 and 10 times more under dynamic loading compared to static loading. Maximum displacements and stresses used in modeling and static and dynamic analyses were close to reality which emphasizes the importance of dynamic analysis of structures.

The results of this paper can be used in ID Fan foundation design of cement plants. By selecting the type of used ID Fan engine and its rotation per minute (rpm) rate, cyclic and dynamic load on the foundation are determined. Then according to the results of this paper, the best type of concrete and rebar in terms of mechanical specifications (Ec, Es) in order to design and construct the foundation with the most lifetime period would be revealed.

\section{References}

[1] P. Nawrotzki, G. Hüffmann, T. Uzunoglu, Static and dynamic analysis of concrete turbine foundations, Structural Engineering International 18(3) (2008) 265270.

[2] C. Rebelo, A. Moura, H. Gervásio, M. Veljkovic, L.S. da Silva, Comparative life cycle assessment of tubular wind towers and foundations-Part 1: Structural design, Engineering Structures 74 (2014) 283-291.

[3] M. Dias Jr, K.L. Cavalca, Experimental analysis of the dynamic behaviour of a turbomachine foundation structure, $r n$ 8(1) (1999) 9.

[4] H. Eskandari, A. Madadi, Investigation of ferrocement channels using experimental and finite element analysis, Engineering Science and Technology, an International Journal 18(4) (2015) 769-775.

[5] M. Zaaijer, Foundation modelling to assess dynamic behaviour of offshore wind turbines, Applied Ocean Research 28(1) (2006) 45-57.

[6] B.T. Cease, Using Vibration Analysis To Identify \& Help Correct An ID Fan Foundation Problem.

[7] R. Thompson, D. Wong, Boiler induced draft fan optimisation, International Sugar Journal 113(1350) (2011) 425-431.

[8] R.J. Sayer, Finite Element Analysis--A Numerical Tool for Machinery Vibration Analysis, Sound and vibration 38(5) (2004) 18-21.

[9] R.J. Sayer, Structural Dynamics of Centrifugal Fans, Proceedings of the National Technical Training
Symposium and 34th Annual Meeting of the Vibration Institute, Oak Brook, IL, 2010.

[10] K. Singleton, B.M. P.E., Case study analysis of centrifugal fan high amplitude vibration

[11] N. Jungbluth, C. Bauer, R. Dones, R. Frischknecht, Life cycle assessment for emerging technologies: case studies for photovoltaic and wind power (11 pp), The International Journal of Life Cycle Assessment 10(1) (2005) 24-34

[12] J. Seo, J.S. Jang, D. Bai, Lifetime and reliability estimation of repairable redundant system subject to periodic alternation, Reliability Engineering \& System Safety 80(2) (2003) 197-204.

[13] S. Deolalkar, Handbook for designing cement plants, (2009).

[14] F. Akgül, D.M. Frangopol, Lifetime performance analysis of existing prestressed concrete bridge superstructures, Journal of Structural Engineering 130(12) (2004) 1889-1903.

[15] F. Akgül, D.M. Frangopol, Lifetime performance analysis of existing reinforced concrete bridges. II: Application, Journal of infrastructure systems 11(2) (2005) 129-141.

[16] H. Eskandari, M.G. Nik, A. Pakzad, Foundation analyzing of centrifugal ID fans in cement plants, Alexandria Engineering Journal (2016).

[17] H. Eskandari-Naddaf, M. Azimi-Pour, Performance evaluation of dry-pressed concrete curbs with variable cement grades by using Taguchi method, Ain Shams Engineering Journal (2016).

[18] H. Eskandari, A. Pakzad, Applying Simplex Lattice In Optimizing Self-Compaction Concrete Compressive Strength, Asian Journal Of Civil Engineering (Bhrc) 16(6) (2015) 775-787.

[19] H. Eskandari-Naddaf, R. Kazemi, ANN prediction of cement mortar compressive strength, influence of cement strength class, Construction and Building Materials 138 (2017) 1-11.

[20] A. Madadi, H. Eskandari-Naddaf, M. Gharouni-Nik, Lightweight Ferrocement Matrix Compressive Behavior: Experiments Versus Finite Element Analysis, Arabian Journal for Science and Engineering 42(9) (2017) 40014013

[21] E. Bognøy, V. Vee, T.M.S. Mo, Fatigue Capacity of partially loaded Areas in Concrete Structures submerged in Water, (2014). 